\title{
La pince optique
}

\author{
Jean-Pierre GALAUP \\ Laboratoire Aimé Cotton - Bât. 505, Centre d'Orsay \\ jean-pierre.galaup@lac.u-psud.fr
}

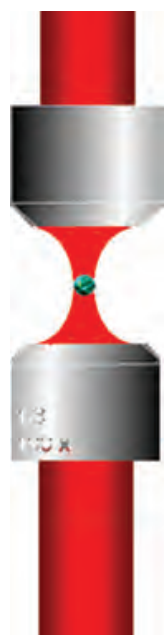

Soupçonné dès le XVIIe siècle avec les observations des queves de comètes toujours orientées face au soleil par Kepler, puis les travaux de Maxwell et une première démonstration expérimentale par Lebedev au XIX' siècle, le fait que la lumière exerce des forces sur la matière, et donc sur des objets est désormais bien établi, mais, considérées comme trop faibles pour être exploitables, ces forces sont restées simple curiosité jusqu'à l'invention du laser dans les années 60 . Dès lors, l'évolution fut rapide et continue grâce aux travaux dans lesquels le nom d'Arthur Ashkin [1] revient souvent : premières réalisations dans les années 70 de pièges optiques à l'aide de deux faisceaux contre-propageant, premières expériences de lévitation optique de microsphères, première pince optique stable à l'aide d'un faisceau laser unique fortement focalisé par un objectif à grande ouverture numérique créant une force optique de gradient en 1986. C'est cette date qu'il convient de garder comme "date de naissance » de la pince optique telle que nous l'exposons dans le présent article et dont nous évoquerons les nombreuses applications.

$\mathrm{L}$ e recours à un faisceau laser focalisé par un objectif de microscope orientait dès lors nombre d'applications potentielles vers la biologie avec des avantages particuliers à cette technique. C'est encore Ashkin qui fut novateur en ce domaine en réalisant les premières manipulations de virus et de bactéries ou d'organites à l'intérieur du cytoplasme cellulaire. D'une part, les pinces optiques (optical tweezers en anglais) permettent la manipulation précise d'objets sans aucun contact avec comme conséquence le fait de pouvoir rester en milieu parfaitement stérile lors de cette manipulation. D'autre part, les forces générées par les pinces optiques sont typiquement équivalentes aux forces mises en jeu dans un grand nombre de processus cellulaires (adhésion, mécanique du cytosquelette, motricité, fonctionnement des moteurs moléculaires...). C'est pourquoi le développement des pinces optiques s'est fait et continue de se faire très majoritairement dans le domaine des applications biologiques. D'autres applications sont également considérées dans les autres domaines de la photochimie ou de la physique comme l'étude et le contrôle de particules colloïdales, de la polymérisation ou de la croissance cristalline ou la mise en mouvement et le contrôle par un faisceau lumineux de micromoteurs ou micropompes. Des pinces optiques peuvent aussi être avantageusement combinées avec d'autres techniques comme la microscopie confocale, la microscopie de fluorescence sous excitation laser à un ou à deux photons, la microscopie Raman. Pour une revue toujours d'actualité de ce domaine, on se référera utilement à la lettre de ressource de Lang et Block [2].

\section{L'action mécanique de la lumière}

La lumière est une onde électromagnétique qui transporte de l'énergie grâce à laquelle le soleil nous chauffe, c'est bien connu mais aussi une quantité de mouvement ou impulsion, grâce à laquelle la lumière exerce une action mécanique et nous pousse un tout petit peu! De cela, il est bien plus difficile de s'en rendre directement compte et sur les objets macroscopiques, cette action de la lumière est le plus souvent ignorée.
Cette action mécanique de la lumière se caractérise par le fait que chaque photon transporte un moment linéaire (c'est la quantité de mouvement ou impulsion déjà évoquée), mais aussi un moment angulaire susceptible d'induire des mouvements de rotation ou vortex. Dans un faisceau de lumière circulairement polarisé, chaque photon transporte un moment angulaire de rotation égal à $\pm \hbar$ selon que la polarisation circulaire est droite ou gauche. C'est le moment angulaire intrinsèque associé au spin du photon. On peut aussi induire un moment angulaire qualifié d'orbital associé à un faisceau. Le front d'onde est alors ordonné et adopte par exemple une structure hélicoïdale comme dans les faisceaux structurés selon un mode dit « Laguerre-Gauss ».

\section{Principe de la pince optique}

La figure $1 a$ illustre schématiquement les éléments essentiels pour construire une pince optique. Ces éléments précisés sur la figure $1 b$ sont : un faisceau laser, un objectif de microscope à grande ouverture numérique, un échantillon contenant 


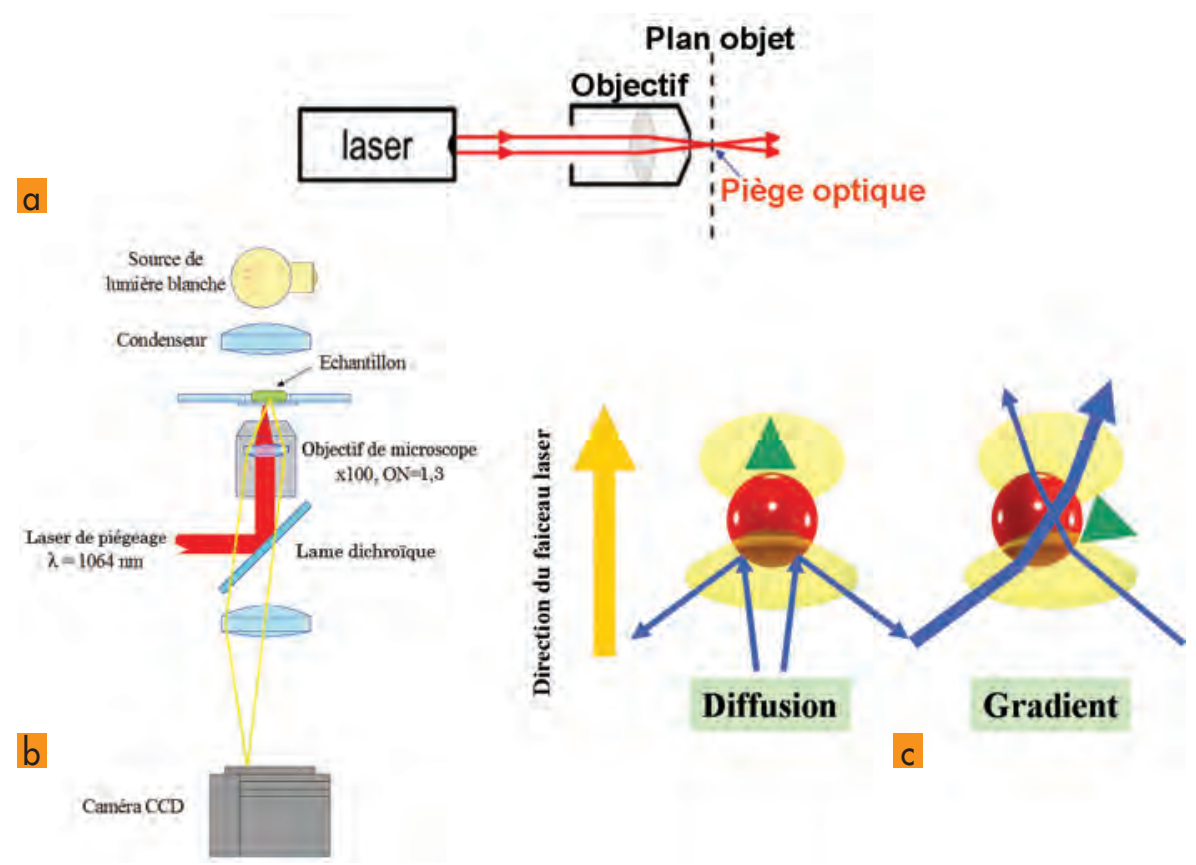

Figure 1. Principe de la pince optique.

les objets à manipuler et un dispositif de visualisation. L'ouverture numérique $(O N)$ est une caractéristique essentielle d'un objectif qui dépend de l'indice de réfraction du milieu interposé entre l'objet et la face d'entrée de l'objectif et de l'angle formé par trois points particuliers : le centre de la lentille, le foyer objet de la lentille et le bord de la lentille. Plus cet angle sera grand, meilleures seront la luminosité (plus de rayons lumineux se trouvent collectés) et la résolution.

Le principe physique de la pince optique générée par la focalisation d'un faisceau laser est illustré à la figure 1c. Pour rendre compte des forces générées au niveau du piège optique, il faut s'appuyer sur la théorie généralisée de Lorenz-Mie qui décrit la diffusion de la lumière par un objet de forme quelconque. En pratique, on distingue deux cas limites selon que la taille de l'objet, ici une bille diélectrique sphérique, a des dimensions plus grandes ou plus petites que la longueur d'onde de la lumière de piégeage. Aux fins d'illustration, nous nous limiterons au cas où l'optique géométrique conserve sa validité. Les microbilles sont des particules sphériques diélectriques transparentes à la lumière incidente et dont l'indice de réfraction est supérieur à celui du milieu ambiant (généralement de l'eau).

Les rayons réfléchis contribuent à la force de diffusion qui pousse l'objet dans la direction du faisceau laser, mais les faisceaux réfractés incidents sous un angle élevé vont " prendre la bille par la taille» et la maintenir au voisinage du point de focalisation, là où l'intensité lumineuse est la plus forte, grâce aux forces de gradient. Le piège est stable dès lors que les forces de gradient surpassent les forces de diffusion.

\section{Exemple de montage expérimental}

Le montage expérimental montré à la figure 2 utilise un laser infrarouge à fibre dopé ytterbium pour le piégeage et conjointement un faisceau laser HeNe pour une évaluation des forces optiques générées au niveau du piège. Détaillons sommairement : le télescope constitué des lentilles L1 et L2 est important pour un bon réglage de l'entrée du faisceau laser dans l'objectif du microscope. Correctement positionnée, la lentille L1 peut être manipulée selon les directions $X, Y$ et $Z$, ce qui garantit un déplacement du piège dans le plan focal objet de l'objectif dans les directions transversales $x$ et $y$ et aussi en profondeur $z$. Une lampe blanche et une caméra CCD permettent la visualisation des objets étudiés sur un écran vidéo.
La mesure de la force du piège optique repose sur l'enregistrement du spectre des fluctuations de lumière du laser $\mathrm{He}-\mathrm{Ne}$ induites par les mouvements perçus par une photodiode à quatre quadrants (PSD) de la bille dans le piège. Les fluctuations sont dues à l'agitation thermique du liquide (mouvement brownien), agitation ample et de basse fréquence si le piège est faible, mais de faible amplitude et de plus haute fréquence si la force du piège augmente.

Aujourd'hui, les dispositifs optiques permettent de créer des pinces multiples avec la possibilité de les contrôler indépendamment les unes des autres, offrant ainsi un contrôle selon les trois dimensions de l'espace. Sous cet aspect, l'introduction des techniques holographiques, notamment des hologrammes de phase, permet de moduler un faisceau laser pour générer pratiquement n'importe quelle distribution spatiale d'intensité souhaitée. Ces travaux introduisirent l'usage des modulateurs spatiaux de lumière qui permettent de créer des structures spectrales complexes en temps réel et d'étendre le nombre de pièges manipulés simultanément à plus de cent !

\section{La pince optique holographique}

Dans un montage de pince holographique tel que montré à la figure 3, un élément essentiel est introduit. Un modulateur spatial de lumière (SLM) à cristaux liquides remplace le miroir $M$ du montage précédent. Le cheminement du faisceau de piégeage est légèrement modifié pour tenir compte du fait que le SLM travaille mieux sous faible incidence. À cause de I'anisotropie des cristaux liquides, il est aussi dépendant de la polarisation de la lumière incidente, d'où l'introduction d'un rotateur de polarisation à rhomboèdres de Fresnel pour l'ajuster au mieux.

Le modulateur à cristaux liquides est pixélisé comme un écran plat de visualisation. Chaque pixel est contrôlé par ordinateur et agit sur la phase de l'onde qui se réfléchit localement sur lui. Il s'agit d'un modulateur de phase qui perme l'inscription d'hologrammes de phase calculés par ordinateur. 


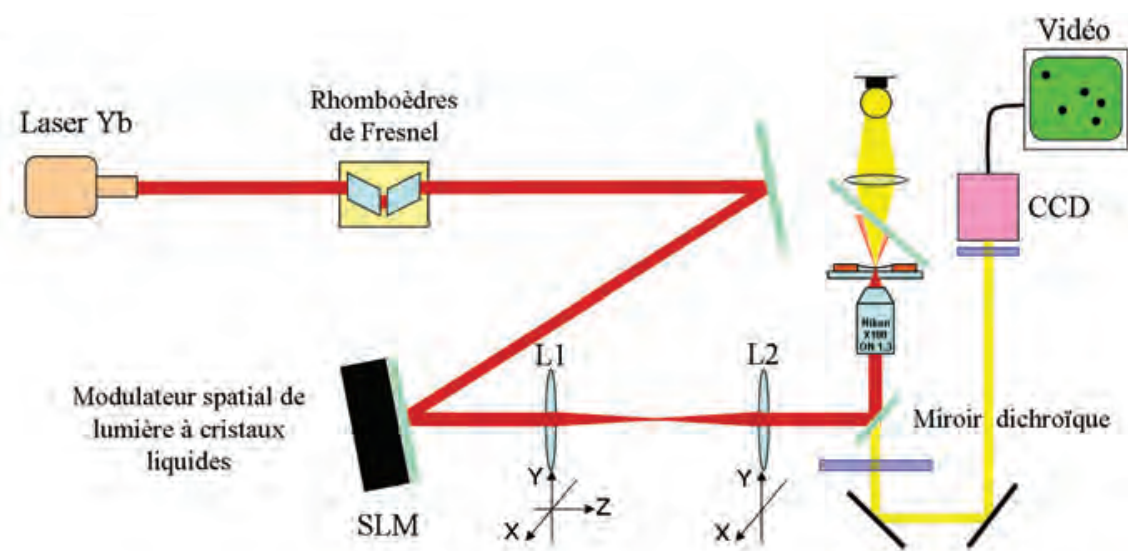

Figure 3. Montage d'une pince optique holographique.

La figure 4 illustre le principe. Sur le SLM on a inscrit un hologramme « à fourche » obtenu en combinant une variation circulaire de la phase de 0 à $2 \pi$ un nombre I de fois autour du pixel central avec un réseau blasé pour séparer les ordres de diffraction de l'ordre 0 . Ainsi, le faisceau diffracté à l'ordre 1 aura les caractéristiques d'un faisceau creux dit de "Laguerre-Gauss » d'ordre $I$. On a reporté à droite quelques exemples avec l'hologramme, la forme prédite du faisceau diffracté (circulaire, carré, triangulaire) et l'enregistrement du faisceau réel obtenu.

Outre la génération de faisceaux avec différentes structures spatiales, on peut générer plusieurs pièges simultanément comme le montre la séquence d'images montrant l'évolution de plusieurs billes au cours du temps pour illustrer les capacités d'une telle méthode.

\section{Quelques applications des pinces optiques}

Les applications des pinces optiques sont très importantes, notamment en biologie: ces pinces s'intègrent facilement dans des systèmes d'imagerie microscopique auxquels elles ajoutent la capacité de manipulation et de contrôle des objets observés. Elles ont l'avantage d'être très flexibles et facilement modulables et programmables, notamment par le recours à I'holographie numérique qui permet de multiplexer le faisceau laser en plusieurs pièges pour manipuler simultanément
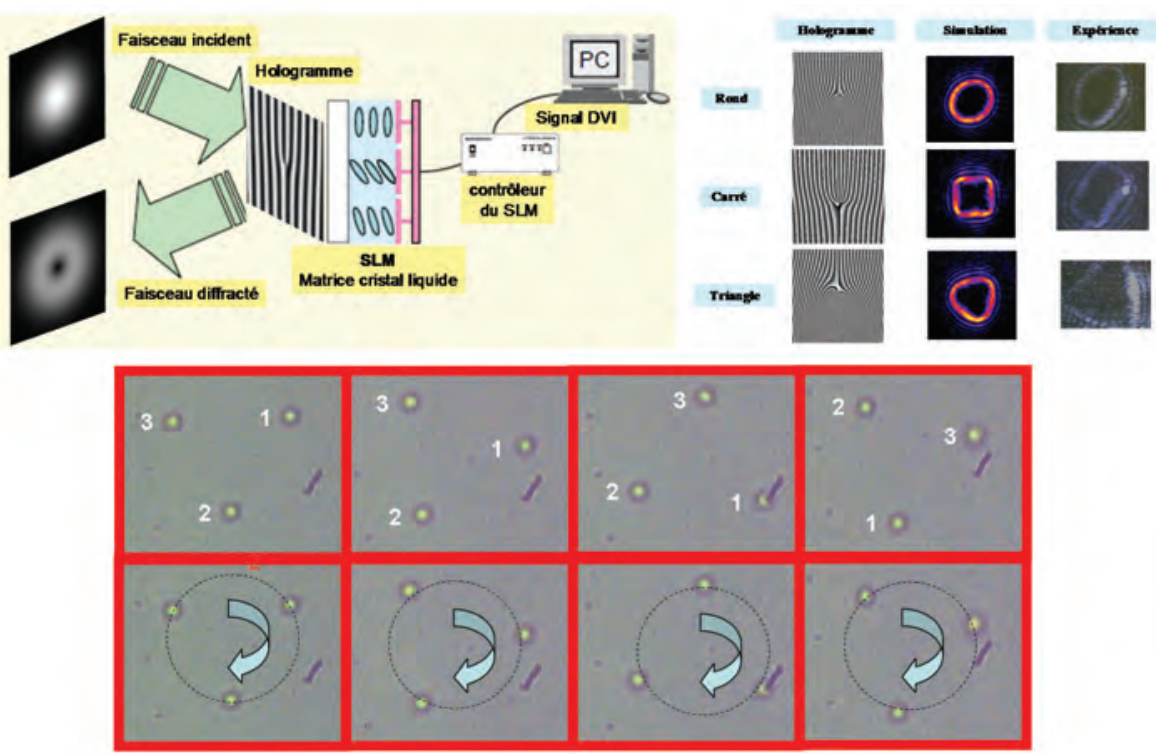

Figure 4. Illustration du principe d'utilisation d'un modulateur spatial de lumière au sein du montage d'une pince optique.

\section{Raman and Fluorescence solutions}

Introducing the new high

performance spectrometers family

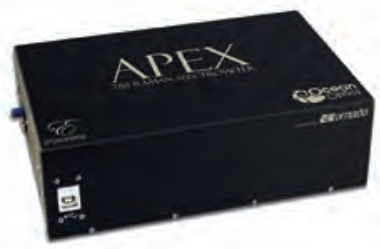

Apex: Sensitivity and Resolution

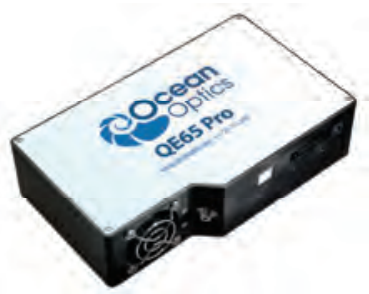

QE65Pro: high SNR and long integration time

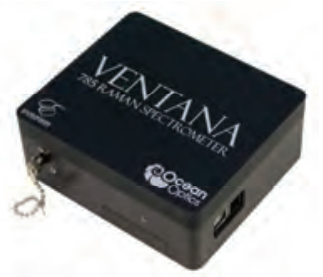

Ventana: Throughput and Sensitivity

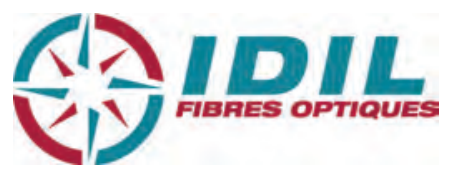

Contact us today to learn more about our complete spectrometer offerings:

T: 0296054020

info@idil.fr | www.idil.fr

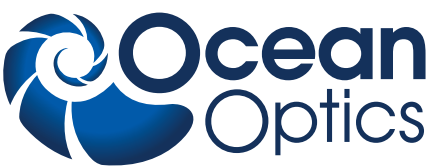

www.oceanoptics.eu 
plusieurs particules. La résolution spatiale est excellente (100 nm à $100 \mathrm{~mm}$ ). Pour les applications biologiques, les lasers recommandés opèrent dans le proche infrarouge, entre 800 et $1064 \mathrm{~nm}$, sous des puissances modérées. Ils sont non-invasifs et évidement stériles, ce qui signifie que leurs effets thermiques ou biologiques sur des cellules vivantes sont quasi nuls. L'autre champ d'application concerne les nano et micro technologies avec particulièrement le contrôle tout optique de dispositifs mécaniques tels que micro-pompes ou micromoteurs.

\section{Molécules individuelles}

Les études dynamiques au niveau des molécules uniques comme les molécules d'ADN ou d'ARN ont grandement progressé grâce aux manipulations à l'aide de pinces optiques. Il est ainsi possible aujourd'hui de mesurer la force appliquée à une molécule d'ADN qui est fixée par l'une de ses extrémités à la surface d'un porte-échantillon et par son autre extrémité à une bille de latex que l'on maintient par une pince optique. On peut ainsi mesurer directement l'élasticité de la molécule d'ADN (figure 5a).

Une autre application a été l'étude de molécules d'ARN polymérase responsables de la transcription du contenu de I'ADN en ARN messager. Pendant la transcription, la molécule d'ARN polymérase se déplace le long du brin d'ADN pour former pas à pas I'ARN messager et il est possible de détecter ce mouvement avec une précision de l'ordre de l'angstrœm en liant une sphère de latex à la molécule d'ARN polymérase (figure $5 b$ ), le déplacement de cette molécule affectant la position de la bille dans le piège optique. La séparation de la double hélice de I'ADN est réalisable à travers l'action d'une force mécanique exercée avec une pince optique. Il a ainsi été possible de déterminer la force avec laquelle sont liées les paires de bases azotées et on a pu établir que ces forces varient selon la séquence de paires de bases.

Une majorité des processus biologiques impliquent la transformation de l'énergie chimique en énergie mécanique au travers de ce qu'on appelle des moteurs moléculaires. Des processus comme le transport intracellulaire, le mouvement de bactéries,
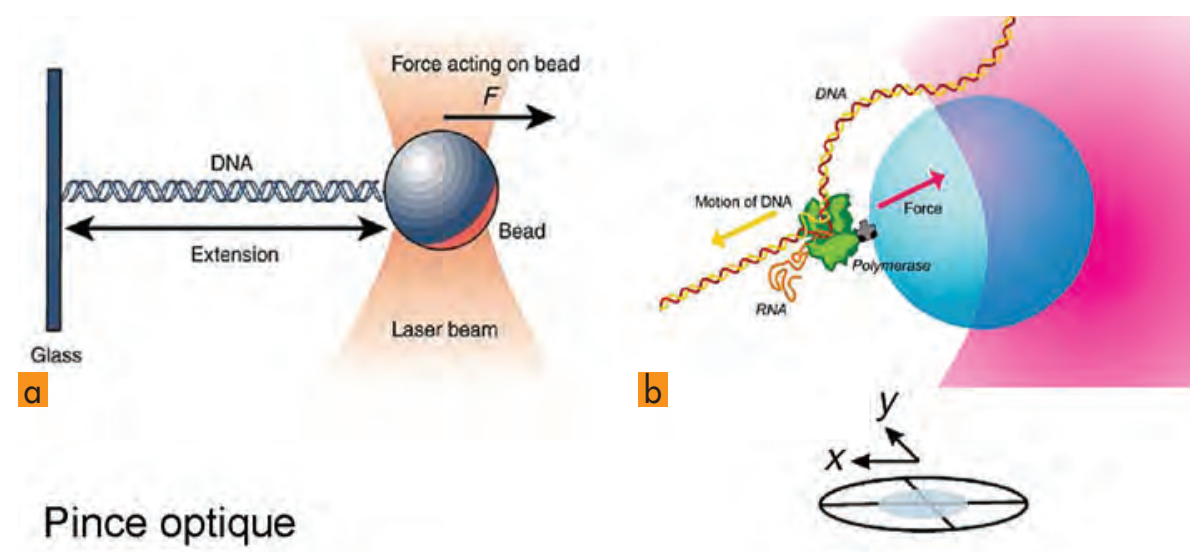

\section{Pince optique}

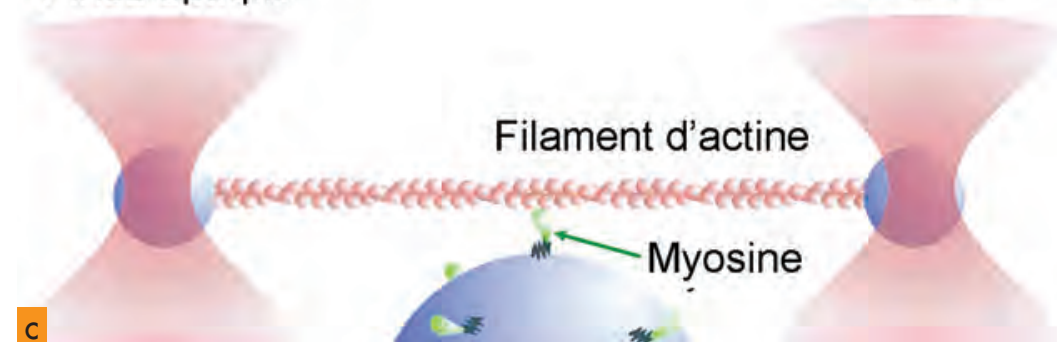

Figure 5. Exemples d'applications des pinces optiques dans l'étude des molécules d'ADN et d'ARN et des moteurs moléculaires.

la réplication de l'ADN ou la contraction musculaire sont des exemples où interviennent différents types de moteurs moléculaires. Les moteurs biologiques sont d'excellents systèmes modèles pour l'étude des mouvements des protéines ou de leurs changements de conformation. De nombreuses études concernent le problème du fonctionnement des fibres musculaires où agissent des molécules comme la kinésine ou la myosine. Un système prototype souvent étudié est constitué d'un filament d'actine attaché à ses extrémités à des billes de latex, ellesmêmes maintenues en position par deux pièges optiques (figure $5 \mathrm{c}$ ). La molécule de myosine interagit avec le filament le long duquel elle peut se déplacer, permettant l'étude des forces en jeu dans la contraction musculaire.

\section{Manipulations de cellules}

C'est un domaine où les expériences sont nombreuses. Grâce au fait que les rayons lumineux traversent aisément les membranes cellulaires, les chromosomes, mitochondries et autres organites intracellulaires peuvent être manipulés par des pinces optiques. Le fonctionnement cellulaire a ainsi fait l'objet de nombreux travaux. En particulier, on relèvera les études portant sur l'influence d'un stress causé à une cellule que l'on a emprisonnée par plusieurs billes venant exercer une pression autour d'elle, sur son métabolisme. D'autres études sur les cellules combinent les pinces optiques avec la microchirurgie laser ou au micro-scalpel, ou encore avec l'utilisation de sondes fluorescentes que l'on vient positionner en des zones précises de la cellule. Une application intéressante concerne l'étude des gamètes et spermatozoïdes et l'aide que les pinces optiques peuvent apporter pour aider à la fertilité humaine ou animale (figure $6 a$ ). La manipulation optique de cellules mobiles comme les spermatozoïdes pour la mesure de leur force de propulsion flagellaire constitue un outil diagnostique de certaines stérilités.

En assurant un contrôle précis des forces exercées sur les membranes des cellules, les pinces optiques ont contribué à une meilleure connaissance de leurs propriétés viscoélastiques. Par exemple, un globule avec deux billes en positions diamétralement opposées est déformé en augmentant la distance entre les pièges (figure 6b). Ainsi, l'étude de l'élasticité de la membrane du globule rouge permet de comprendre comment l'absence ou l'anomalie de certaines protéines membranaires peut entraîner une déformation persistante du globule susceptible 


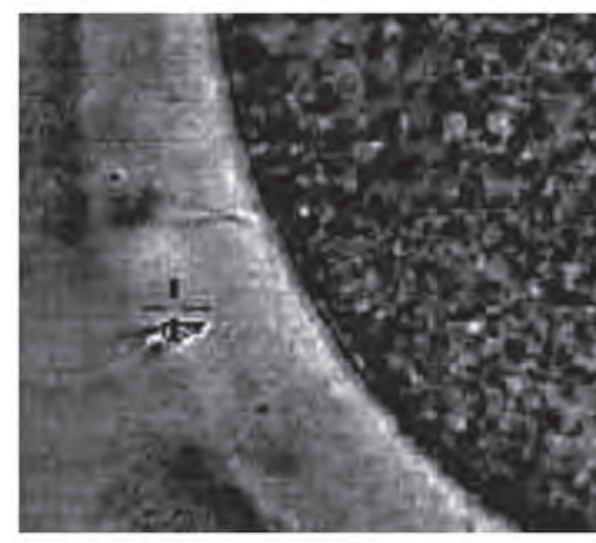

Aide à la fertilisation

Mesure de viscoélasticité

\section{a}

b

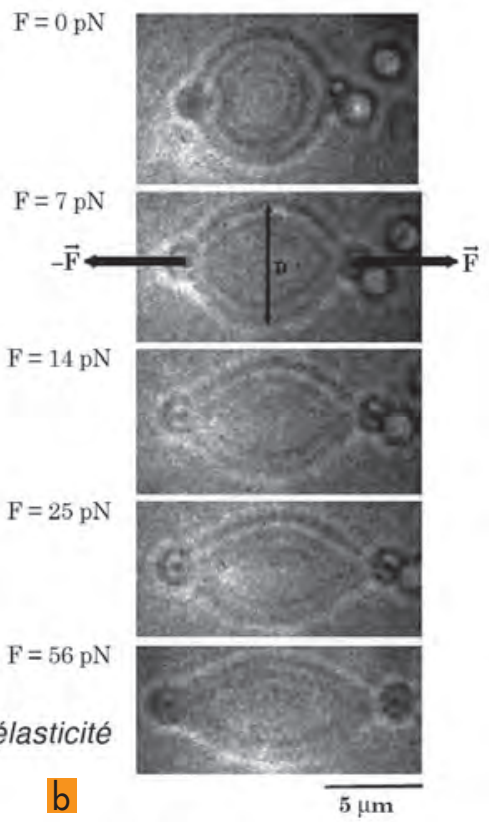

Figure 6. Exemples d'utilisation de pinces optiques dans le domaine de la manipulation des cellules.

de favoriser sa destruction prématurée. On peut aussi étudier les propriétés viscoélastiques de protéines ou de chaînes peptidiques, la flexibilité et la résistance à la torsion de brins d'ADN, la sélection et la mise en contact de cellules photosensibles avec des cellules neuronales pour analyser le développement des contacts intercellulaires et la régénération de synapses entre ces deux types cellulaires.

\section{Applications non-biologiques: nano et micromoteurs}

Un autre grand domaine d'applications concerne l'étude et la fabrication de nano- ou de micromoteurs, un thème de recherches où beaucoup de travaux portent sur les différentes possibilités de mettre en rotation par la lumière de petits objets et de contrôler leur vitesse et sens de rotation. Les applications diverses micro-fluidique : moteurs de circulation, agitateurs, vannes, viscosimètres locaux... L'idée motrice de ces recherches en micro fluidique souvent combinées avec l'utilisation de pinces optiques est la réalisation de micro-laboratoires ou lab-on-a-chip. Dans la compréhension de systèmes colloïdaux, cette technique a déjà démontré sa grande utilité pour étudier leur comportement dynamique et les interactions entre particules colloïdales.

La figure 7 présente deux exemples de réalisations. À gauche, des micro-rotors sont animés par le faisceau laser d'une pince optique : le rotor se met à tourner au voisinage du foyer de l'objectif et le sens de rotation change selon que le rotor est au dessus ou au-dessous du foyer. Au centre, on illustre une application en micro fluidique : dans un micro canal en envisagées concernent notamment la
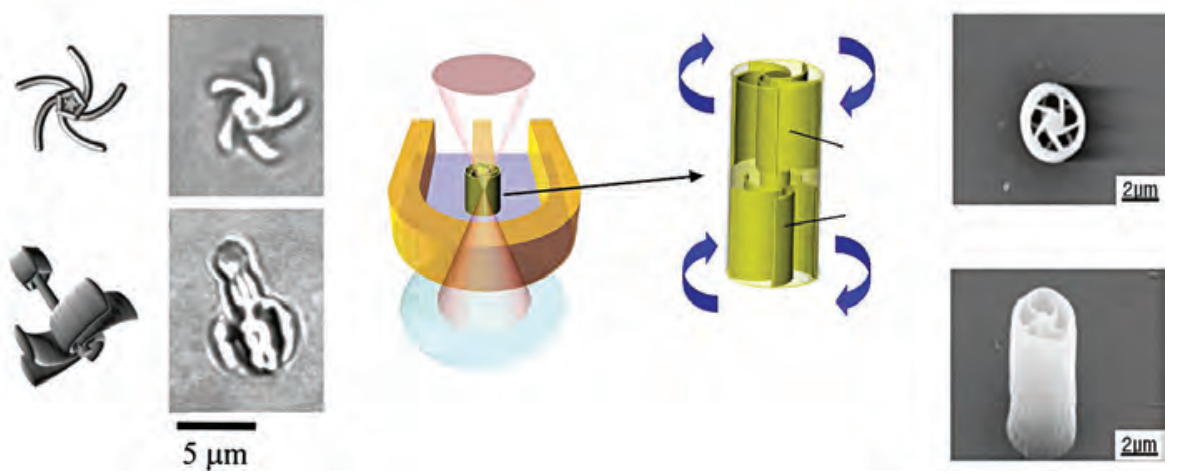

Figure 7. Exemples de réalisations non biologiques utilisant des pinces optiques.

U est placée une micro pompe dont la réalisation pratique est montrée à droite.

\section{Un thème en pleine évolution}

Il est difficile de conclure sur un thème toujours en pleine évolution, tant dans les applications que dans le développement technologique. Voici néanmoins quelques pistes pour illustrer ce foisonnement.

\section{La pince avec retour d'effort : pour une sensation tactile de la micromanipulation}

Pour l'étude et la réalisation de microcomposants, particulièrement difficiles et coûteuses, la micro-robotique propose des solutions automatiques ou télé opérées. Des interfaces de micromanipulation haptique (c'est-à-dire avec retour d'effort) à pince optique sont développées, permettant à l'utilisateur de « sentir » l'effort qu'il exerce.

\section{La pince au bout des doigts}

La figure 8 présente une tablette iPad utilisée comme interface entre la pince optique physique et l'utilisateur (J. Opt. 13 (2011) 044022).

\section{Au-delà de la pince...}

L'action de la lumière ne se limite pas à la capacité à capturer un objet. D'autres situations sont explorées comme la possibilité de faire « voler » un objet en forme d'aile d'avion, le flux de photons assurant la portance requise, ou celles jouant sur des configurations particulières de faisceau conduisant à ce que, selon leur taille où leur indice, certaines particules soient attirées et d'autres repoussées par la lumière, réalisant un triage automatique et efficace d'un mélange d'objets.

\begin{tabular}{l} 
Bibliographie: \\
[1] A. Ashkin, "History of optical trapping and \\
manipulation of small neutral particles, atoms \\
and molecules" in Single Molecule Spectroscopy, \\
R. Rigler, M. Orrit, and T. Basché, eds. (Springer, \\
2001), pp. 1-31. \\
[2] M. J. Lang and S. M. Block, "Ressource letter: \\
LBOT-1: laser based optical tweezers", Am. J. \\
Phys. 71 (2003) 201. \\
[3] D. McGloin, "Optical tweezers : 20 years \\
on", Phil. Trans. R. Soc. A 364 (2006) 3521. \\
\hline
\end{tabular}

